Polymer Journal Vol. 1, No. 3, pp. 348-355 (1970)

\title{
Gel Formation and Structure of Junctions in Poly(Vinyl Alcohol)-Water Systems
}

\author{
Kyoichiro Shibatani \\ Central Research Laboratories, Kurashiki Rayon Company, \\ Sakazu, Kurashiki, Okayama, Japan.
}

(Received January 30, 1970)

\begin{abstract}
The main purpose of this investigation was to elucidate the mechanism of gelation of poly(vinyl alcohol)-water systems and the structure of junctions in gel networks. Changes of gel rigidities were measured as a function of gelling time at the temperatures of $30-80^{\circ} \mathrm{C}$ for four samples of different tacticities, and the numbers of junctions in the gel were calculated using the theory of rubber elasticity. The formation of junctions can be treated as the binary association of free cross-linking sequences with the activation energy of $13 \mathrm{kcal} / \mathrm{mol}$. The study of the correlation between gelling temperatures and melting temperatures of gels shows that the junctions formed at a given temperature should be treated as multitudinous in structure and the copolymeric character of atactic poly(vinyl alcohol) chains should be considered. The structures of junctions for various gelling temperatures are discussed, assuming that cross-linking loci in poly(vinyl alcohol) chains are syndiotactic sequences of different sequence lengths.

KEY WORDS: Poly(Vinyl Alcohol) / Aqueous Solution / Gelation /

Kinetics / Network / Junction / Tacticity / Syndiotatic Sequence /
\end{abstract}

An aqueous solution of poly(vinyl alcohol) (PVA) is well known to form a thermally reversible gel upon standing at low temperature. Diversity of opinion has persisted, however, concerning the mechanism of the gelation and the structure of junction points in a gel network. The appearance of an X-ray crystalline diffraction pattern has been reported for a filament gel spun from a very concentrated solution $^{1}$ and micellar structure has been suggested for junctions. ${ }^{2}$ The heat of gelation has been estimated to be $8.8 \mathrm{kcal} / \mathrm{mol}^{3}$ from the dependence of the melting temperature of PVA gel on the polymer concentration using an equation derived by Eldridge and Ferry, ${ }^{4}$ and junctions have been ascribed to weak interactions of some hydroxyl groups. ${ }^{3}$ The significant retardation of the gelation is observed by the introduction of a very small amount of comonomer units in a PVA. ${ }^{5-7}$ This observation leads to the opinion that the gelation of PVA aqueous solution results from intermolecular bondings of a substantially large number of hydroxyl groups in a chain. ${ }^{6}$

Flory and Garrett have shown that the gelations of collagen systems occur as a consequence of a crystal-liquid phase transformation ${ }^{8}$ of a small fraction of chain units. The copolymeric character of the molecule is conducive to gel formation, for even if the equilibrium requirements are fulfilled, all the chain units would not participate in the junction, so that only a small fraction of them would be transformed. ${ }^{9}$ Conventional PVA, which is atactic in structure, possesses a copolymeric character, and the structure of junctions seems to be multitudinous in size and in stereochemical configuration. Consequently, the application of Eldridge and Ferry's equation to PVA-water systems seems to be unsuitable, for the equation assumed that the cross-links can be treated as single in structure, all with the same free energy of association.

The PVA-water gel exhibits rubber elasticity ${ }^{10}$ and the network structure is stable on deformation, unlike PVA-water-borate gel, of which the chemical relaxation time at room temperature has been reported to be in the order of $0.3 \mathrm{sec}^{11}$ In the investigation reported in this paper, the rigidity and equilibrium swelling volume of gels have been measured as a function of gelling period and temperature, using lightly cross-linked samples which bear repeated 
gelling and melting on a dynamometer. The number $\nu$ of network chains per unit volume of polymer has been obtained by the following equation given by Flory for swollen fibrous proteins $^{12}$

$$
\tau=k T \nu\langle\alpha\rangle_{0}^{2} v_{2}^{-1 / 3}\left(\alpha-\alpha^{-2}\right)
$$

where $\tau$ is the retractive force at an extension ratio $\alpha$ which is the ratio of the length of the deformed sample to that of the undeformed swollen sample, and $v_{2}$ is the volume fraction of the polymer in the swollen sample. The dilation factor $\langle\alpha\rangle_{0}$ is given by $\langle\alpha\rangle_{0}=\left(\bar{r}^{2} / \bar{r}_{0}\right)^{1 / 2} v_{2}{ }^{1 / 3}$ and the ratio of the mean square end-to-end distance $\bar{r}^{2}$ of the network chain to that of the free chain $\bar{r}_{0}{ }^{2}$ is assumed to be unity in isothermal gelling processes. The mechanism of gelation and the configuration of PVA chains which form junctions will be also discussed in this paper.

\section{EXPERIMENTAL}

\section{Materials}

A sample of PVA was obtained by hydrolysis of poly(vinyl formate) prepared by free-radical polymerization of its monomer with the catalyst of $\mathrm{B}\left(\mathrm{C}_{2} \mathrm{H}_{5}\right)_{3}-\mathrm{O}_{2}$ at $-78^{\circ} \mathrm{C}$. Three PVA samples were prepared by cationic polymerization (catalyst, $\left.\mathrm{BF}_{3} \cdot \mathrm{O}\left(\mathrm{C}_{2} \mathrm{H}_{5}\right)_{2}\right)$ of vinyl $t$-butyl ether at $-78^{\circ} \mathrm{C}$ in the mixtures of toluene and methylene chloride of different ratios. Details of the preparative conditions are described elsewhere. ${ }^{13}$ Table I lists the values of triad-tacticity estimated from the NMR spectra of 10- $\%$ solutions of acetylated samples ${ }^{14,15}$ in methylene chloride measured by a Varian HA-100 spectrometer at $60^{\circ} \mathrm{C}$. The value of 1,2-glycol content measured by polaro- graphy $^{16}$ and the number-average molecular weight $\bar{M}_{n}$ of PVA samples estimated from a calibrated GPC curve are also given in Table I.

\section{Samples}

Hot solutions of 10-\% PVA samples in water were cast into glass plates and were slightly irradiated by electron beams (EBG $2000 \mathrm{KVP}$, General Electric Co., U.S.A.) at ca. $90^{\circ} \mathrm{C}$. Rod-like strips $(1 \times 2 \times 30 \mathrm{~mm})$ were cut from the thus obtained lightly cross-linked gels and were heated repeatedly with water at $130^{\circ} \mathrm{C}$ in sealed glass tubes to remove soluble fractions. In consequence, samples were obtained which could be used in the gelling and melting experiments repeatedly without any change.

\section{Stress-Strain Measurement}

The dynamometer for the stress and strain measurements was essentially the same as that used in the previous investigation. ${ }^{17}$ A schematic diagram of the apparatus is shown in Figure 1.

At the beginning of a series of experiments the temperature of water in which the sample was immersed was kept at ca. $98^{\circ} \mathrm{C}$ for more than 2 hours to dissolve all thermally reversible junctions. In the case of the sample BE-1, a mixture of 20-\% n-propanol and $80-\%$ water was employed to dissolve the junctions completely and the solution was replaced repeatedly by boiling water. The inner cell of the apparatus was thereupon filled with water whose temperature was controlled at gelling temperature within $\pm 0.1^{\circ} \mathrm{C}$ during the gelation of samples by a water jacket thermostated by a Model FSe thermostat (Haake Co., W.G.), and stress-strain measurements or force measurements at constant sample length were started. The temperature

Table I. Four kinds of PVA samples

\begin{tabular}{|c|c|c|c|c|c|c|c|}
\hline \multirow{2}{*}{ Sample } & \multirow{2}{*}{ Monomer } & \multirow{2}{*}{$\begin{array}{l}\text { Polymerization } \\
\text { solvent }^{\mathrm{a}}\end{array}$} & \multirow{2}{*}{$\bar{M}_{n} \times 10^{-4}$} & \multirow{2}{*}{$\begin{array}{l}\text { 1,2-Glycol } \\
(\mathrm{mol} \%)\end{array}$} & \multicolumn{3}{|c|}{ Tacticity } \\
\hline & & & & & $I$ & $H$ & $S$ \\
\hline $\mathrm{F}$ & Vinyl formate & Methyl formate & 4.40 & 0.3 & 17.0 & 46.0 & 37.0 \\
\hline BE-1 & Vinyl $t$-butyl ether & $\mathrm{M}$ & 4.40 & 0.1 & 16.7 & 48.6 & 34.7 \\
\hline BE-2 & " & M90/T10 & 4.31 & 0.1 & 25.5 & 47.1 & 27.4 \\
\hline BE-3 & $" \prime$ & M80/T20 & 4.75 & 0.2 & 30.7 & 43.7 & 25.6 \\
\hline
\end{tabular}

a $\mathrm{M}$, methylene chloride; $\mathrm{T}$, toluene. 


\section{K. Shibatani}

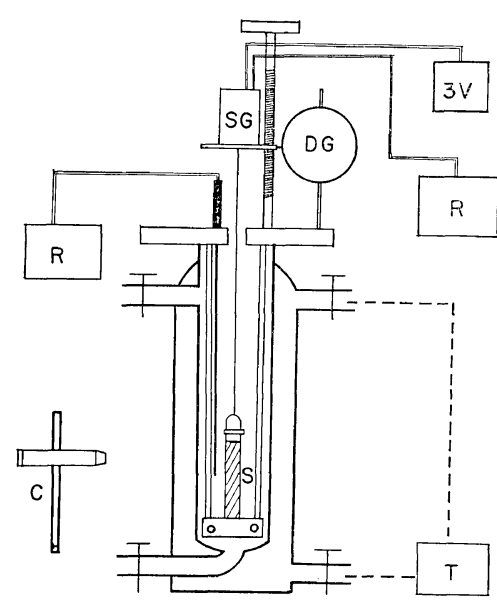

Figure 1. Schematic diagram of dynamometer: S, sample; $\mathrm{SG}$, strain gauge; $\mathrm{DG}$, dial gauge; $\mathrm{R}$, automatic recorder; $\mathrm{T}$, circulating thermostat; $\mathrm{C}$, cathetometer.

of the water in which the sample was immersed was recorded continuously by a Model ER-10 automatic recorder (Yokogawa Electric Co.) or by a Type $3072 \mathrm{X}-\mathrm{Y}$ recorder (Yokogawa). Transducers used for stress measurements were a Model UL-10-120 (Shinko Co.) and Green Cell (Statham Co., U.S.A.) operating with an input voltage of 3 volts. The output voltage was recorded by an $\mathrm{X}-\mathrm{Y}$ recorder or by a Model QPD 54 recorder (Hitachi). These transducers were frequently calibrated by a weight. Stressstrain measurements at constant temperatures were carried out by changing the strain stepwise four or five times after the stress at each strain appeared approximately constant. The measurements took about 30 minutes per sample at the initial stage of the gelling process and about 2 hours per sample at the advanced stage of gelation.

The measured tensions were referred to the cross-section of the dried sample. The volume fraction $v_{2}$ of polymer in the swollen gel was calculated from the sample length $L_{i}$ at zero force assuming that the sample was isotropic, namely, $v_{2}{ }^{1 / 3}=L_{i 0} v_{20}^{1 / 3} / L_{i}$, where $L_{i 0}$ stands for the sample length at zero force at a known degree of swelling $v_{20}$, which is given by the ratio of the weight of swollen gel to that of dried gel.

\section{RESULTS}

Force Measurements and Properties of Gel

The retractive force to keep a sample strip at a constant length during the whole process of gelation is illustrated as a function of the time for different gelling temperatures in Figure 2 for sample $\mathrm{F}$ cross-linked by the irradiation of $2 \mathrm{Mrad}$. It is seen that the gelation of PVA in water is a slow process. The force increased rather rapidly at higher temperatures, but the equilibrium force seems to be smaller for higher gelling temperatures. After the force became nearly constant, the temperature of water in which gel was immersed was increased at a rate of $1.7^{\circ} \mathrm{C} / \mathrm{min}$. Increasing the temperature, the force increased at first and then fell rapidly as shown in Figure 3. The melting process of the gel was very rapid compared with the gelling process. The overlap of the melting curves for different gelling temperatures seems to suggest that the gels in melting are in a quasiequilibrium

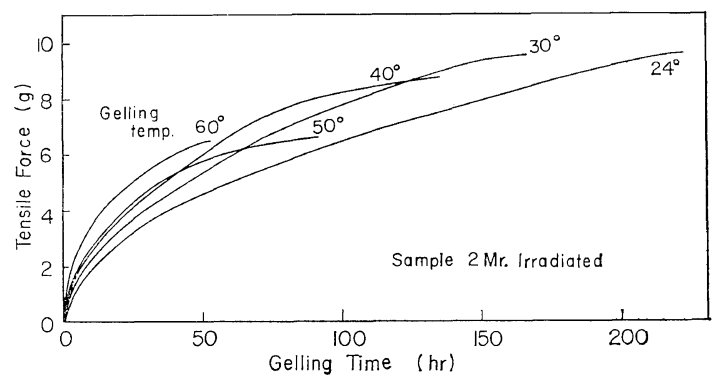

Figure 2. Change of retractive force with gelling time for sample of F-PVA.

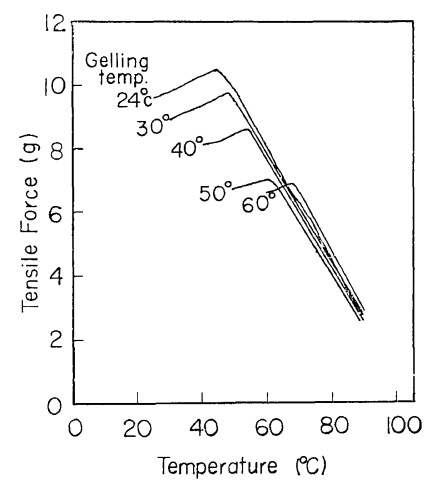

Figure 3. Variation of force with elevation of temperature for gelled sample shown in Figure 2. 
state. The temperature $T_{m}$, at which the force attains the maximum value and $(\partial \ln \tau / \partial T)_{P, L}=0$, namely,

$$
\begin{aligned}
(\partial \ln \nu / \partial T)_{P, L}= & -1 / T_{m}+\beta_{\mathrm{Sw}} /\left(\alpha^{3}-1\right) \\
& -\beta_{\mathrm{d}} / 3+d \ln r_{0}{ }^{2} / d T
\end{aligned}
$$

may be assumed as the temperature at which junction points in gel begin to melt. Here $\beta_{\mathrm{SW}}$ and $\beta_{\mathrm{d}}$ stand for the the thermal expansion factors of the swollen gel and dried PVA respectively. The observed value of $T_{m}$ was independent of the rates of increasing temperature from 0.7 to $2.0^{\circ} \mathrm{C} / \mathrm{min}$ and of the periods of gelation from 24 to 200 hours, though hot water resistance of PVA gel has been reported to increase with gelling period. ${ }^{1} \quad T_{m}$ values were plotted against the temperature $T_{x}$ of gelation in Figure 4 for three samples of F-PVA irradiated at different doses. Relations between $T_{x}$ and $T_{m}$ may give the equilibrium melting temperature $\left(90^{\circ} \mathrm{C}\right)$ of these gels, though some deviation from linearity were observed at higher temperatures of gelation.

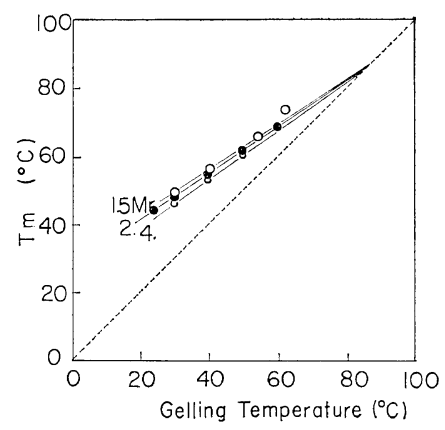

Figure 4. Plots of gelling temperature vs. $T_{m}$ of gel for F-PVA samples of irradiation dose 4,2 and 1.5 Mrad.

For the sample initially gelled at $30^{\circ} \mathrm{C}$ for one month in a free, unstretched condition, the force at $10 \%$ extension and the degree of swelling $v_{2}$ were observed at four different temperatures. The temperature was increased stepwise. From the data listed in Table II, it is seen that some of the junctions in the gel melt by increasing the temperature, but new junctions are formed slowly by standing the gel at a constant temperature. These findings suggest that junctions formed at a certain temperature are multi-
Table II. Change of $v_{2}$ and rigidity of gel ${ }^{\mathrm{a}}$ with stepwise increase of temperature

\begin{tabular}{lrrr}
\hline $\begin{array}{c}\text { Tempera- } \\
\text { ture } \\
\left({ }^{\circ} \mathrm{C}\right)\end{array}$ & $\begin{array}{c}\text { Time after elevation } \\
\text { of } \begin{array}{c}\text { temperature } \\
(\mathrm{hr})\end{array}\end{array}$ & $v_{2}$ & $\tau$ at $\begin{array}{c}\alpha=1.1 \\
(\mathrm{~g})\end{array}$ \\
\hline 30.0 & - & 0.081 & 11.25 \\
\hline 44.3 & 2 & 0.108 & 8.53 \\
& 6 & 0.081 & 9.40 \\
& 22 & 0.110 & 10.40 \\
\hline 58.3 & 2 & 0.111 & 8.60 \\
& 6 & 0.113 & 9.05 \\
& 24 & 0.117 & 10.48 \\
\hline 73.5 & 2 & 0.118 & 8.90 \\
& 24 & 0.122 & 9.95 \\
\hline 98.0 & 2 & 0.048 & 2.90 \\
\hline
\end{tabular}

a Sample, 2 Mrad irradiated F-PVA.

tudinous in structure.

Rigidity Change with Time and Polymer Stereoregularity

Four kinds of cross-linked specimens obtained from PVA samples of different stereoregularities by the irradiation of 1.5 Mard were used for the isothermal gelation experiments in free, unstretched conditions at $30-80^{\circ} \mathrm{C}$. The elastic modulus and $v_{2}$ were measured as a function of gelling time. Values of $\nu$ were calculated from Eq. 1. Typical examples of the change in $v_{2}$ with gelling time are shown in Figure 5 for FPVA samples. As gelation proceeds, the change in $v_{2}$ becomes rather small and $v_{2}$ stays nearly 0.2 .

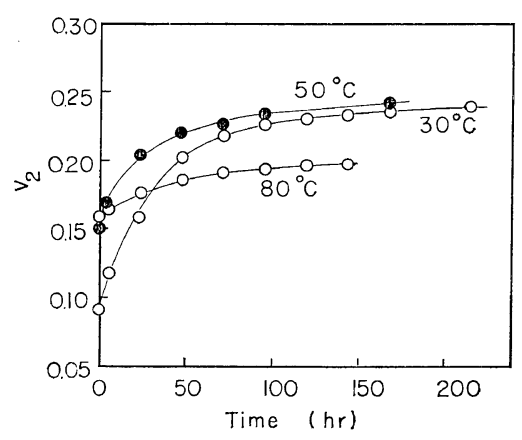

Figure 5. Change of $v_{2}$ by gelling time for F-PVA sample. 


\section{K. Shibatani}

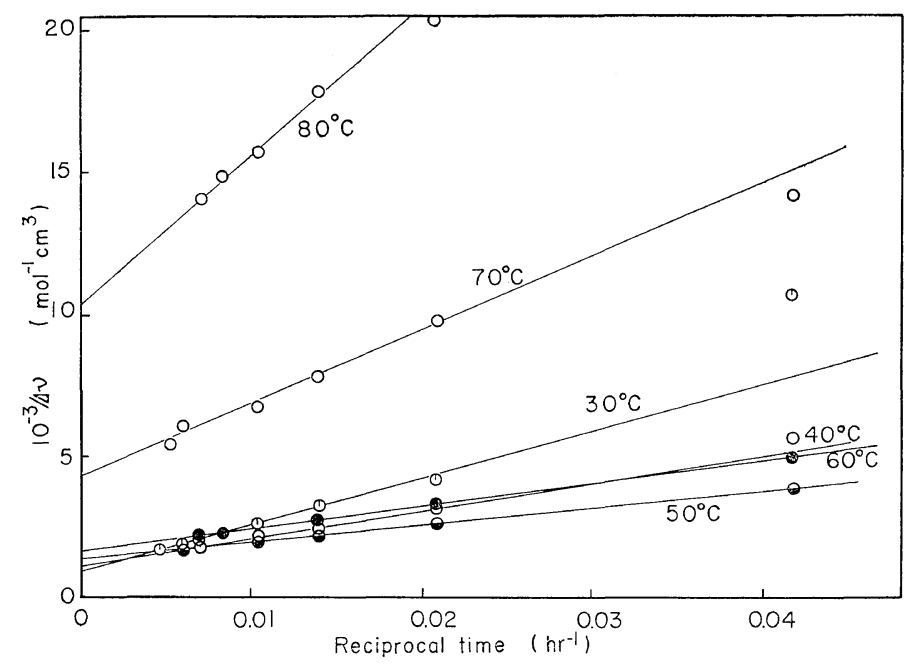

Figure 6. Reciprocal number of network chains plotted against reciprocal gelation time for F-PVA samples.

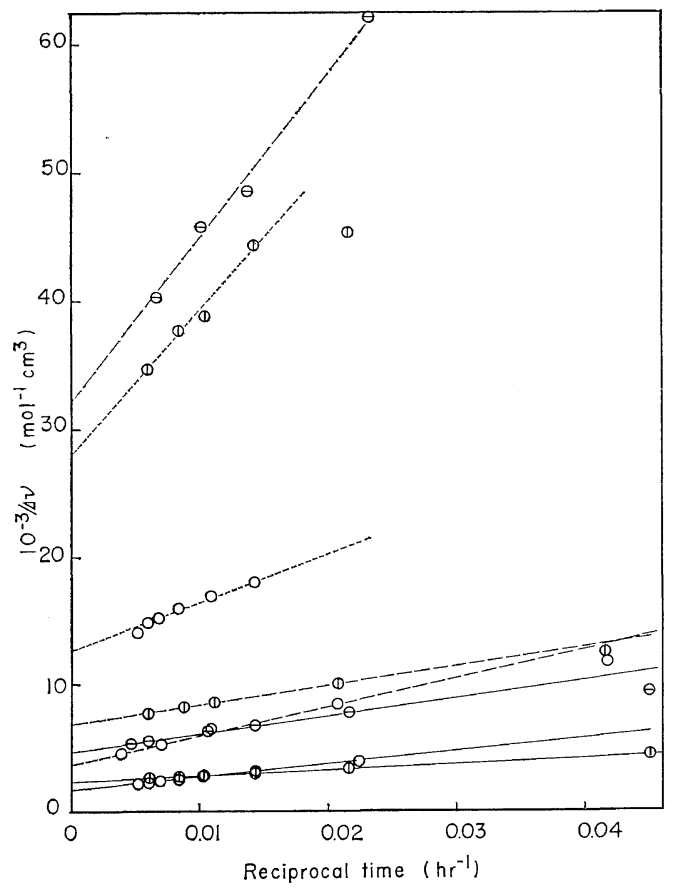

Figure 7. Plots of $1 / \Delta \nu$ against reciprocal gelling time for samples of PVA type: -, BE-1; --, BE-2; ---, BE-3 (temperature of gelation: $\mathrm{O}, 30^{\circ} \mathrm{C} ; \mathbb{1}$, $50^{\circ} \mathrm{C} ; \ominus, 70^{\circ} \mathrm{C}$.

The number $\Delta \nu$ of network chains formed by gelation is obtained by

$$
\Delta \nu=\nu-\nu_{t=0}
$$

where $\nu_{t=0}$ is the number of network chains formed by the irradiation of electron beams. The reciprocal of $\Delta \nu$ is plotted against the reciprocal gelling time in Figure 6. It is seen that the gelation proceeds most rapidly and most extensively at $50^{\circ} \mathrm{C}$ for a gelling period of less than 100 hours for this sample. Extrapolating the linear relation to $1 / t=0$, we may get the number $\Delta \nu_{t=\infty}$ of chains in gel networks at an infinite gelling time for various temperatures of gelation. The linearity between $1 / \Delta \nu$ and reciprocal gelling time is also the case for the other specimens as is seen in Figure 7. The equilibrium concentrations of the gel specimens were ca. $20 \%$ for the sample obtained from BE-1 PVA and ca. $15 \%$ for the sample from BE-2 PVA. The aqueous solution of BE-3 PVA hardly forms gel, and the equilibrium concentration of the specimen from this sample was only $2 \%$. The values of $\Delta \nu_{t=\infty}$ for PVA samples of different stereoregularities at various temperatures are listed in Table III.

\section{DISCUSSION}

It has often been reported ${ }^{18-20}$ that each crosslink in gel networks may be treated as formed by binary associations between chains. Assuming that junctions are formed by binary associations of all of the cross-linking loci, the rate 
Table III. Gradients and intercepts of lines in Figures 6 and 7, and sequence lengths of syndiotactic units in a junction calculated from Eq. 7

\begin{tabular}{lcccccc}
\hline Sample & $\begin{array}{c}\text { Temperature } \\
\left({ }^{\circ} \mathrm{C}\right)\end{array}$ & $\begin{array}{c}a=\Delta \nu t=\infty \\
\left(10^{4} \times \mathrm{mol}^{-} \mathrm{cm}^{-3}\right)\end{array}$ & $\begin{array}{c}\text { Gradients of lines in } \\
\text { Figures } 6 \text { and } 7 \\
\left(10^{-5} \times \mathrm{mol}^{-1} \cdot \mathrm{cm}^{3} \cdot \mathrm{hr}\right)\end{array}$ & $n$ & $\begin{array}{c}\Delta \nu_{t=\infty}^{\prime} \\
\left(10^{4} \times \mathrm{mol} \cdot \mathrm{cm}^{-3}\right)\end{array}$ & $n^{\prime}$ \\
\hline F & 30 & 11.07 & 1.65 & 4.89 & 11.18 & 4.86 \\
& 40 & 8.98 & 0.964 & 5.32 & 9.07 & 5.30 \\
& 50 & 7.37 & 0.594 & 5.73 & 7.44 & 5.71 \\
& 60 & 5.93 & 0.768 & 6.17 & 5.44 & 6.36 \\
& 70 & 2.37 & 2.60 & 8.05 & 3.25 & 7.42 \\
& 80 & 0.98 & 5.30 & 9.90 & 1.23 & 9.44 \\
\hline BE-1 & 30 & 6.17 & 1.05 & 5.56 & 8.01 & 5.07 \\
& 50 & 4.13 & 0.44 & 6.32 & 4.92 & 5.99 \\
& 70 & 2.11 & 1.44 & 7.58 & 2.57 & 7.21 \\
\hline BE-2 & 30 & 2.69 & 2.18 & 6.38 & 4.01 & 5.74 \\
& 50 & 1.48 & 1.50 & 7.35 & 2.20 & 6.71 \\
& 70 & 0.32 & 12.7 & 9.81 & 0.62 & 8.75 \\
\hline BE-3 & 30 & 0.80 & 3.80 & 8.49 & & \\
& 50 & 0.36 & 11.1 & 9.78 & & \\
\hline
\end{tabular}

of formation of junctions is written as

$$
d x / d t=k(a-2 x)^{2}
$$

Here $a$ is the concentration of cross-linking loci at a gelling temperature, and $x$ is the concentration of junctions at time $t$. The coefficient $k$ is the rate constant of the gelling reaction. Integration of Eq. 4 gives

$$
1 / x=1 / k a^{2} t+2 / a
$$

The linear relations between $1 / \Delta \nu$ and $1 / t$ in Figures 6 and 7 suggest that assumptions of binary association are permissible. Thus, we can get a concentration of cross-linking loci in PVA from the intercept at $1 / t=0$ and the rate constant of gelation from the gradient.

In the preceding section, it has been pointed out that the structure of cross-linking sequences at a certain temperature is multitudinous. The fact that the association of cross-linking loci of different structures is treated with a single rate constant may indicate that the formation of nucleus is the rate-determining step in the gelaintion process.

$$
2 \text { (free cross-linking loci) } \stackrel{k}{\rightarrow} \text { nucleus } \rightarrow \text { cross-link }
$$

The rate constants of the gelation for all samples are plotted in Figure 8 as a function of temperature. The effect of $v_{2}$ on the gela-

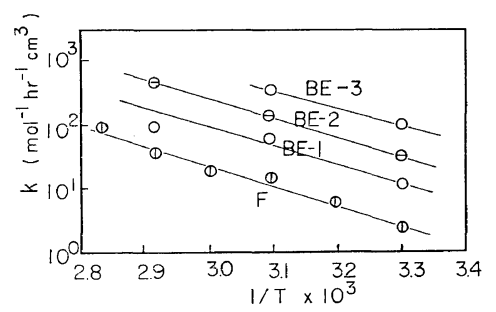

Fjgure 8. Rate constant of gelation plotted against reciprocal absolute temperature, for specimens obtained from PVA samples of different stereoregularities.

tion would be included in $k$, but the difference of $k$ values among samples could not be completely explained. The activation energy of the gelation is estimated as $13 \mathrm{kcal} / \mathrm{mol}$ using the Arrhenius equation.

As is obvious from Table III, the gelling property of PVA in water is highly promoted by a slight increase of syndiotacticity. Isotactic diol parts in PVA are inclined to form intramolecular hydrogen bonds, ${ }^{21-22}$ and isotactic PVA is known to dissolve easily in cold water, while syndiotactic samples would not dissolve even in boiling water. Syndiotactic parts in PVA chains in water would be inclined to form intermolecular junctions. It seems reasonable, therefore, to assume that the cross-linking loci 


\section{K. Shibatani}

are syndiotactic sequences whose sequence lengths are larger than a critical value $n$, and that these sequences randomly associate to form junctions without distinguishing their sequence lengths from each other.

The number of moles of the sequence $S_{n-1}$ in a cubic centimeter whose sequence lengths are not more than $n-1$ may be written as

$$
\begin{aligned}
S_{n-1}= & d p(1-p)^{2}\left\{1+(P / p)+(P / p)^{2}+\cdots\right. \\
& \left.+(P / p)^{n-2}\right\} / M_{0} \\
= & d p^{2}(1-p)^{2}\left\{1-(P / p)^{n-1}\right\} /\left(M_{0} p-P\right)
\end{aligned}
$$

where $P$ and $p$ are the syndiotacticities in triad and in dyad ( $p=S+H / 2)$, respectively, $M_{0}$ is the molecular weight of the monomer unit and $d$ is the density of the sample $(d=1.27)$. Thus the number of moles $S_{n}$ of sequences whose sequence lengths are greater than or equal to $n$ is

$$
S_{n}=d p^{2} P^{n-1}(1-p)^{2} / M_{0} p^{n-1}(p-P)
$$

Substitution of $\Delta \nu_{t=\infty}$ values shown in Table III into $S_{n}$ 's gives $n$ 's for the corresponding temperatures. The values of $n$ are listed in the fifth column of Table III. The agreement of $n$ 's for a gelling temperature of samples of different tacticities is not satisfactory. A possible reason for this disagreement is that Eq. 1 is insufficient when applied to gel networks ${ }^{23-25}$ formed in solution because of wasted loop formations, small cycle formations, chain entanglements and so on. Jackson and Gill ${ }^{26}$ have measured the elastic modulus of gels formed by the reaction of known amounts of terephthalaldehyde with PVA samples of various molecuar weights in water, and have shown that the topological factor, which is defined as the experimentally obtained elastic modulus divided by $\nu k T$ calculated from the amount of reacted terephthalaldehyde, is a linear function of polymer concentrations for each sample. We have examined the modulus of gels obtained by the reaction of known amounts of glyoxal with aqueous solutions of PVA having the numberaverage molecular weight of $44 \times 10^{3}$, and have found $\Gamma=0.03$ at $c=4.42$ and $\Gamma=0.17$ at $c=8.24$, where $\Gamma$ is the ratio of the calculated $\nu$ value to the observed $\nu$ value and $c$ is the concentration $(\mathrm{g} / \mathrm{d} l)$ of PVA. Consequently we may have

$$
\Gamma=0.035 c-0.08 \quad(c>2.2)
$$

The numbers of gel network chains, $\Delta \nu_{t=\infty}$ in this work are divided by $\Gamma$ and the corrected numbers $\Delta \nu_{t=\infty}^{\prime}$ of network chains are estimated. As is seen in the seventh column of Table III, $n^{\prime}$ values obtained by the substitutions of $\Delta \nu_{t=\infty}^{\prime}$ values into Eq. 7 show fairly good agreement among samples of different stereoregularities. This fact seems to support the values in Table III and the treatment that cross-linking loci in PVA solutions are syndiotactic sequences.

Acknowledgement. The author wishes to thank Dr. M. Matsumoto for his continued encouragement and Drs. Y. Oyanagi and K. Katsuura for their helpful discussions during the work.

\section{REFERENCES}

1. Y. Sone, K. Hirabayashi, and I. Sakurada, Kobunshi Kagaku (Chem. High Polymers), 10, 1 (1953).

2. N. Hirai, Nippon Kagaku Zasshi (J. Chem. Soc. Japan, Pure Chem. Sect.), 75, 697 (1954).

3. H. Maeda, T. Kawai, and R. Kashiwagi, Kobunshi Kagaku (Chem. High Polymers), 13, 193. (1956).

4. J. E. Eldridge and J. D. Ferry, J. Phys. Chem., 58, 992 (1954).

5. T. Kominami, R. Naito, and Y. Odanaka, Kobunshi Kagaku (Chem. High Polymers), 12, 218 (1955).

6. G. Takahashi and I. Sakurada, ibid., 13, 502. (1956).

7. S. A. Glikman, L. A. Roth, L. I. Khomutov, E. N. Gubenkova, and I. I. Feigelson, J. Polym. Sci., Part C, 16, 2001 (1967).

8. P. J. Flory and R. R. Garrett, J. Amer. Chem. Soc., 80, 4836 (1958).

9. L. Mandelkern, "Crystallization of Polymers," McGraw-Hill, New York, N.Y., 1964, p 113.

10. N. Hirai, Nippon Kagaku Zasshi (J. Chem. Soc. Japon, Pure Chem. Sect.), 74, 539 (1953); 75. 695 (1954).

11. R. K. Schultz and R. R. Myers, Macromolecules, 2, 281 (1969).

12. P. J. Flory, J. Amer. Chem. Soc., 78, 5222 (1956).

13. K. Shibatani, M. Nakamura, and Y. Oyanagi, Kobunshi Kagaku (Chem. High Polymers), 26, 118 (1969). 
14. K. Fujii, Y. Fujiwara, and S. Fujiwara, Makromol. Chem., 89, 278 (1965).

15. S. Murahashi, S. Nozakura, M. Sumi, H. Yuki, and K. Hatada, J. Polym. Sci., Part B, 4, 65 (1966).

16. S. Imoto, J. Ukida, and T. Kominami, Kobunshi Kagaku (Chem. High Polymers), 14, 214 (1957).

17. I. Sakurada, A. Nakajima, and K. Shibatani, Makromol Chem., 87, 103 (1965).

18. J. D. Ferry, J. Amer. Chem. Soc., 70, 2244 (1948).

19. N. Hirai, Nippon Kagaku Zasshi (J. Chem. Soc. Japan, Pure Chem. Sect.), 74, 235 (1953); 72, 837 (1951).
20. Y. Oyanagi and M. Matsumoto, Kobunshi Kagaku (Chem. High Polymers), 15, 348 (1958).

21. E. Nagai, S. Kuribayashi, M. Shiraki, and M. Ukita, J. Polym. Sci., 35, 295 (1959).

22. T. Shimanouchi and M. Oka, Preprint (Physics), 12th Symposium on Polymer Chemistry, Nagoya, 1963, p 323.

23. M. J. Voorn and J. J. Hermans, J. Polym. Sci., 35, 113 (1959).

24. W. G. Lloyd and T. Alfrey, ibid., 62, 301 (1962).

25. B. Mukherji and W. Prins, ibid., Part A, 2, 4367 (1964).

26. J. F. Jackson and S. J. Gill, ibid., Part A-2, 5, 663 (1967). 